\title{
THE
}

8-15-1994

\section{Alkali Core-Level Binding-Energy Shifts in Alkali/4d-Metal Interface Systems}

\author{
E. Lundgren \\ M. Qvarford \\ R. Nyholm \\ J. N. Andersen \\ David R. Heskett \\ University of Rhode Island, dheskett@uri.edu
}

Follow this and additional works at: https://digitalcommons.uri.edu/phys_facpubs

Terms of Use

All rights reserved under copyright.

\section{Citation/Publisher Attribution}

Lundgren, E., Qvarford, M., Nyholm, R., Andersen, J. N., \& Heskett, D. (1994). Alkali core-level bindingenergy shifts in alkali/4d-metal interface systems. Physical Review B, 50(7), 4711-4717. doi: 10.1103/ PhysRevB.50.4711

Available at: http://dx.doi.org/10.1103/PhysRevB.50.4711

This Article is brought to you for free and open access by the Physics at DigitalCommons@URI. It has been accepted for inclusion in Physics Faculty Publications by an authorized administrator of DigitalCommons@URI. For more information, please contact digitalcommons-group@uri.edu. 


\title{
Alkali core-level binding-energy shifts in alkali/4d-metal interface systems
}

\author{
E. Lundgren, M. Qvarford, R. Nyholm, and J. N. Andersen \\ Department of Synchrotron Radiation Research, Institute of Physics, Lund University, Solvegatan 14, S-223 62 Lund, Sweden \\ D. Heskett \\ Department of Physics, University of Rhode Island, Kingston, Rhode Island 02881
}

(Received 17 February 1994)

\begin{abstract}
Photoemission from different alkali core levels ( $\mathrm{Na} 2 p, \mathrm{~K} 3 p, \mathrm{Rb} 4 p$, and $\mathrm{Cs} 4 d$ ) has been studied for thin alkali films deposited on four different $4 d$ metal surfaces: $M o(110), \operatorname{Rh}(111), \operatorname{Rh}(110)$, and $\operatorname{Pd}(100)$. Alkali atoms at the interface, the bulk, and at the surface of the adsorbed alkali film are found to have different core-level binding energies. It is found that the core-level binding-energy shifts of the alkali metals induced by the $4 d$-metal substrate increase with increasing atomic number of the alkali metal and with increasing atomic number of the $4 d$ metal. Thermodynamical quantities such as interface segregation energies and adhesion energies are deduced from the layer-resolved shifts. Estimates of the experimental binding-energy shifts are given using semiempirical calculations, and certain complications in doing that are discussed.
\end{abstract}

\section{INTRODUCTION}

The adsorption of alkali-metal atoms on metal surfaces has received much interest from both experimentalists and theorists. ${ }^{1-8}$ One reason for this is that alkali-metal adsorption is in general thought to be a very simple adsorption system, even though several recent investigations $^{2-7}$ indicate that this is not always the case. We have in earlier work made extensive use of highresolution core-level photoemission applied to the substrate and alkali core levels to investigate the geometrical structure of alkali overlayers adsorbed on a metal substrate. ${ }^{2,4,6}$ Another aspect of core-level photoemission concerns how the magnitude of the alkali core-level binding-energy shifts is related to the electronic structure of the surroundings of the alkali adsorbate. The emphasis of the present paper is mainly on that aspect of core-level photoemission.

The ideal system in which to study the core-level binding-energy shift that a metal induces in an alkali metal would be to have a dilute solution of the alkali metal in the metal in question. In practice, however, such a solution is not possible. Instead we have chosen to investigate the interface between a thin alkali film and a metal substrate. In such a system the alkali atoms at the interface, in the intermediate layers (hereafter denoted as bulk atoms), and at the surface of the alkali film have different surroundings. For example, the layer of interface atoms has on one side substrate atoms and on the other side alkali atoms whereas the bulk atoms are completely surrounded by alkali atoms. Thus the interface and the bulk alkali atoms have a relatively well-defined difference in surroundings, yielding different core-level binding energies, although the lack of knowledge of the exact geometrical structure of the interface introduces an uncertainty. Finally it should be noted that similar measurements of core-level binding-energy changes have been made for a number of different interface systems, not necessarily us- ing alkali metals as the adsorbate. ${ }^{9-18}$

In this paper we present layer-resolved core-level binding-energy shifts for $\mathrm{Na}, \mathrm{K}, \mathrm{Rb}$, and $\mathrm{Cs}$ films on the $4 d$ metal surfaces Mo(110), $\mathrm{Rh}(111), \mathrm{Rh}(110)$, and $\operatorname{Pd}(100)$ with the aim of providing detailed experimental information on the trends in the core-level bindingenergy shifts observed in these alkali-4d-metal interface systems. Layer-resolved core-level photoemission from $\mathrm{Na}, \mathrm{K}$, and $\mathrm{Cs}$ on $\mathrm{Ru}(100)$ has been reported by others. ${ }^{13,14}$ In a recent publication we have presented layerresolved core-level binding-energy shifts for $\mathrm{Na}, \mathrm{K}, \mathrm{Rb}$, and Cs on Al(111) (Ref. 16) which demonstrate a trend in the binding-energy shifts of the alkali core levels on an $s p$-metal substrate.

It has been shown elsewhere ${ }^{9}$ that values for thermodynamical quantities may be derived from layer-resolved core-level binding-energy shifts and that conversely these binding-energy shifts may be estimated from thermodynamical parameters. It is demonstrated that in the present systems applications of these models give rise to some complications due to the large size difference between an alkali-metal atom and the next element in the Periodic Table. It is discussed how these complications may be overcome, and procedures are suggested which lead to good agreement with the experimental values.

\section{EXPERIMENT}

The measurements were performed at beam line 22 (Refs. 19 and 20) at the MAX-I storage ring in Lund, Sweden. After cycles of $\mathrm{Ar}^{+}$sputtering followed by annealing, the remaining carbon on the $M o(110), R h(111)$, $\mathrm{Rh}(110)$, and $\mathrm{Pd}(100)$ surfaces was removed by several annealings at $5 \times 10^{-9}$ Torr in oxygen. This treatment produced surfaces which exhibited low-energy electron diffraction patterns with sharp spots and a low background. The cleanliness of the surfaces and of the deposited alkali layers was checked by monitoring the core- 
level regions of possible contaminants, in particular $\mathrm{C}$ and $\mathrm{O}$, indicating no surface contamination during deposition of the alkali films or during acquisition of the spectra. The base pressure in the experimental chamber was $6 \times 10^{-11}$ Torr. The different alkali metals were deposited from well outgassed commercial SAES getter sources. ${ }^{21}$ During evaporation of the alkali metals the pressure increase was $1 \times 10^{-10}$ Torr or less. The photon energies used for the $\mathrm{Na} 2 p, \mathrm{~K} 3 p, \mathrm{Rb} 4 p$, and the Cs $4 d$ spectra were $90,110,39$, and $110 \mathrm{eV}$, respectively. The total energy resolution was in all cases below $100 \mathrm{meV}$. All measurements were made at $90 \mathrm{~K}$ in order to reduce phonon broadening. ${ }^{22,23}$

\section{RESULTS}

When depositing alkali overlayers at $90 \mathrm{~K}$ no signs were observed of intermixing or alloy formation between the adsorbed alkali metal and the $4 d$ metal substrates studied in this paper. As described in more detail below it is straightforward to follow the growth of the alkali films because of the large binding-energy shifts between alkali atoms at the interface, in the bulk, and at the surface of the films. The large binding-energy shifts between emission from the first and the second alkali layers make it very easy to detect when the second layer starts growing. We define one alkali layer as the amount deposited just before the emission from the second layer becomes visible. The alkali coverages quoted below are simply obtained by dividing the evaporation time by the time needed for deposition of one layer. By monitoring the intensities of the alkali core-level peaks from the various layers during growth of an alkali film we find that the first alkali layer is completed before the second layer starts growing, and that the third layer starts to grow before the second layer is completed. In other words, we find a classic Stranski-Krastanov growth mode. This growth mode makes the morphology of the alkali film more complicated than for layer-by-layer growth. This more complicated morphology does, however, not interfere with the present measurements as it is still possible to identify from which layer a given core-level peak originates and thereby determine the layer resolved shifts.

In order to illustrate the general development of alkali core-level photoemission spectra during growth of an alkali film, Fig. 1 shows the $\mathrm{Na} 2 p$ core-level spectra for $\mathrm{Na}$ deposited on $\operatorname{Pd}(100)$ with coverages ranging from approximately one layer to four and a half layers. The onelayer situation yields a broad peak which contains the spin-orbit-split $\mathrm{Na} 2 p$ level, where the spin-orbit splitting is approximately $0.15 \mathrm{eV}$. The strong broadening of this peak is discussed below. Further deposition of $\mathrm{Na}$ results in the appearance of a second $\mathrm{Na} 2 p$ spin-orbit doublet shifted by about $0.8 \mathrm{eV}$ towards higher binding energy. When the $\mathrm{Na}$ coverage is further increased, the high binding-energy $\mathrm{Na} 2 p$ peak grows in intensity while the low binding-energy peak decreases and shifts towards lower binding energy. This development of the intensities shows that the high and low binding-energy peaks can be attributed to the second and the interface layers, respectively. The shift towards lower binding energy of the

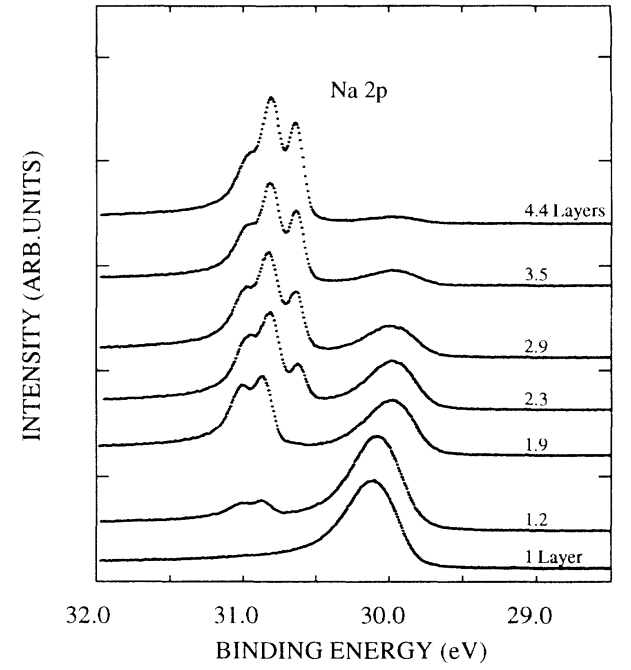

FIG. 1. Na $2 p$ photoemission spectra for coverages from one to approximately four layers of $\mathrm{Na}$ on $\mathrm{Pd}(100)$. The spectra are not normalized in intensity.

first-layer peak between the one- and two-layer situations is due to the fact that after the deposition of the second layer the first alkali layer is no longer a surface layer. This results in a binding-energy shift corresponding roughly to the negative of the surface core-level shift, which is a shift of approximately $-0.20 \mathrm{eV}$. Upon completion of the second $\mathrm{Na}$ layer, the total shift between the first-layer peak, which is now due to emission from the interface $\mathrm{Na}$ atoms, and the second-layer peak, which is due to surface $\mathrm{Na}$ atoms, is approximately $1 \mathrm{eV}$. As the third layer starts growing a third spin-orbit-split doublet appears at a binding energy in between the two peaks already discussed. This peak is associated with the $\mathrm{Na}$ atoms between the interface and the surface atoms which we denote as bulk atoms. The binding energy of this peak is very close to that of the bulk peak from a thick $\mathrm{Na}$ film. Further increase of the $\mathrm{Na}$ coverage does not lead to any additional significant binding-energy shifts of the three different components of the $\mathrm{Na} 2 p$ level, but only to a decrease in the intensity of the interface peak, which disappears gradually as the $\mathrm{Na}$ film grows thicker, and to an increase in the intensity of the bulk peak until it reaches an intensity corresponding to the intensity for a bulk peak from a thick $\mathrm{Na}$ film. The development of the alkali core-level photoemission spectra with coverage as seen in Fig. 1 and as described above, is generally observed for systems which involve $\mathrm{Na}, \mathrm{K}, \mathrm{Rb}$, or Cs deposited on a metal substrate at $100 \mathrm{~K}$, see, for example, Refs. 10 and $13-18$.

In order to demonstrate how the binding-energy shifts vary among different alkalis adsorbed on the same substrate, we show in Fig. 2 the $\mathrm{Na} 2 p, \mathrm{~K} 3 p, \mathrm{Rb} 4 p_{3 / 2}$, and Cs $4 d_{5 / 2}$ core-level spectra of approximately three layers of each alkali metal deposited on $\mathrm{Rh}(111)$. The $\mathrm{Na} 2 p$ and the $\mathrm{K} 3 p$ spectra consist of three spin-orbit-split components. The larger spin-orbit splitting of the $\mathrm{Rb} 4 p(0.85$ $\mathrm{eV})$ and the Cs $4 d(2.3 \mathrm{eV})$ levels allows complete separa- 


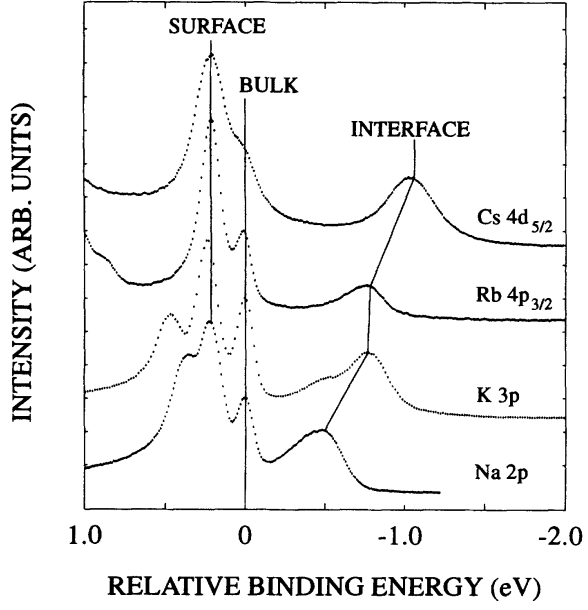

FIG. 2. Core-level spectra for three layers of the indicated alkali metals on $\mathbf{R h}(111)$.

tion of their doublet components, which results in three single peaks in the energy window shown for these spectra. The spectra in Fig. 2 have been aligned so that the bulk binding energies of the four alkali metals coincide. It can be seen from Fig. 2 that the surface core-level shift stays almost constant whereas the binding-energy shift between the interface and the bulk atoms increases as one goes from $\mathrm{Na}$ to $\mathrm{K}$ to $\mathrm{Rb}$ to Cs. This trend is seen for all the substrates investigated in the present work. A qualitatively similar trend has been observed in Refs. 13 and 14 for alkali deposition on $\mathrm{Ru}(100)$ and in Refs. 16-18 for alkali deposition on $s p$-metal substrates.

Turning to the alkali core-level binding energies observed when varying the $4 d$ metal substrate, we present in Fig. 3(a) core-level spectra of approximately three layers of Cs deposited on $\mathrm{Mo}(110), \operatorname{Rh}(111)$, and $\mathrm{Pd}(100)$ as well as from a thick Cs film. In the case of a thick film of Cs

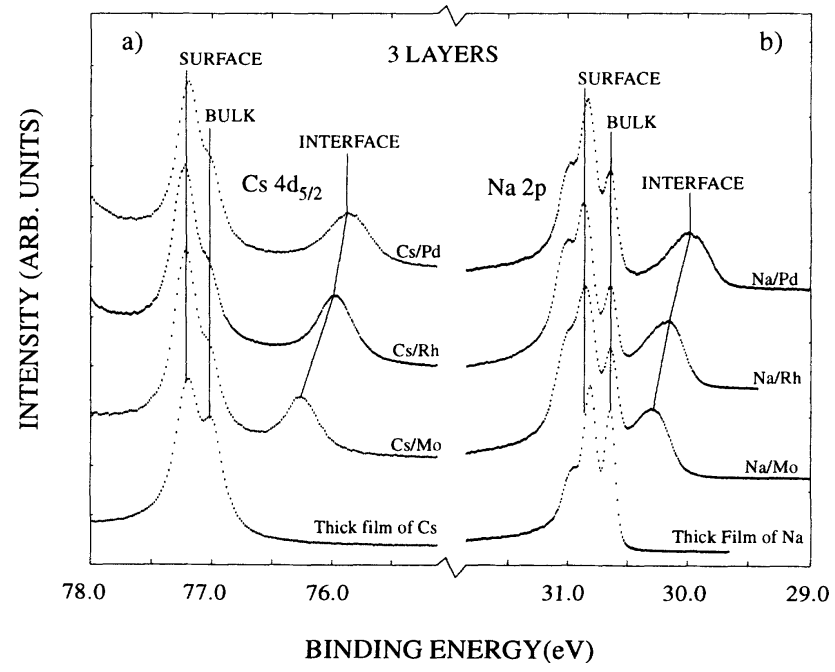

FIG. 3. (a) Cs $4 d_{5 / 2}$ spectra from approximately three layers of $\mathrm{Cs}$ on $\mathrm{Mo}(100), \mathrm{Rh}(111)$, and $\mathrm{Pd}(100)$. (b) $\mathrm{Na} 2 p$ spectra from approximately three layers of $\mathrm{Na}$ on $\mathrm{Mo}(100), \mathrm{Rh}(111)$, and $\operatorname{Pd}(100)$. there is only emission from the atoms in the bulk and the atoms at the surface of the Cs film. It is clear from Fig. (3a) that the binding-energy shift of the Cs $4 d_{5 / 2}$ level between the interface atoms and the bulk atoms increases as the substrate is changed from $\mathrm{Mo}(110)$ to $\mathrm{Rh}(111)$ to $\operatorname{Pd}(100)$, while the surface core-level shift of Cs stays constant. The same qualitative trend is also seen in Fig. 3(b) in which corresponding core-level spectra for the $\mathrm{Na} 2 p$ level are shown. The greater core-level binding-energy shift between the interface and bulk atoms in the Cs case in Fig. 3(a) compared to the $\mathrm{Na}$ case in Fig. 3(b) further illustrates the trend presented in Fig. 2 above.

Whereas the trends discussed above may be seen directly from the raw spectra, a more precise determination of the various core-level shifts requires a decomposition procedure. Figure 4 shows, as an example, a decomposition of the three-layer spectrum of $\mathrm{Na} / \mathrm{Pd}(100)$. The three separated doublets in this fit are indicated and correspond to the peaks mentioned above. It is interesting to note that the interface peak is substantially broader than the bulk and surface peaks. A combination of a number of effects contributes to this extra broadening of the interface peak. First, as the core-level binding energy of adsorbed alkali atoms depends on the exact adsorption site (see, e.g., Refs. 2 and 6), the existence of multiple sites in the interface layer, i.e., the fact that the overlayer is not epitaxial, may give an additional broadening. Similarly other kinds of disorder specific to the interface may cause additional broadening. ${ }^{9,22}$ Second, the vibrational broadening is increased for interface atoms. That this type of broadening gives a significant contribution may be seen from the fact that the width is temperature dependent. ${ }^{22,23}$ Third, the lifetime of an alkali core hole may be shorter for an interface than for a bulk atom which would increase the width of the interface core levels.

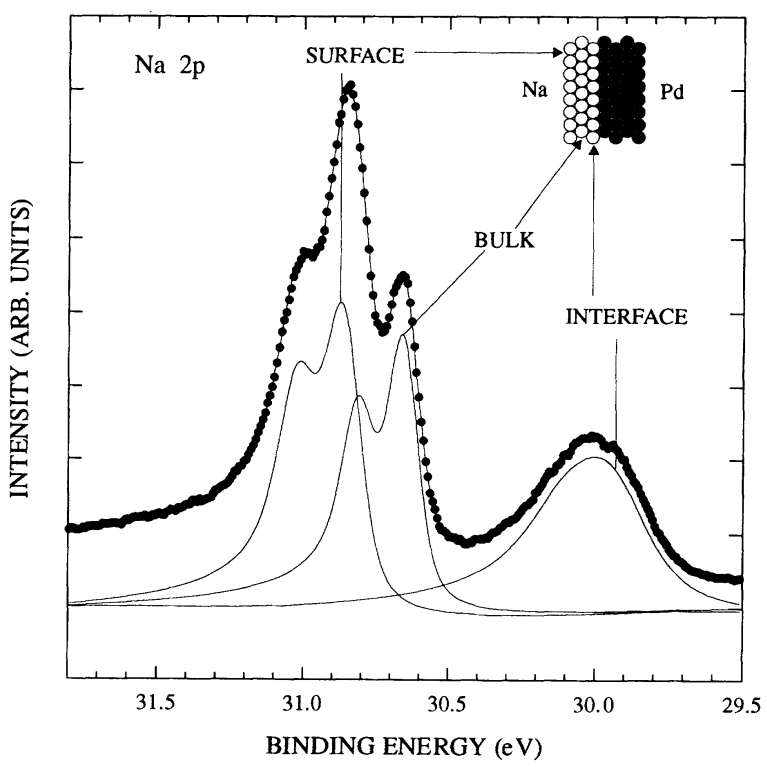

FIG. 4. The decomposition of the $\mathrm{Na} 2 p$ spectrum for $\mathrm{Na}$ deposited on $\mathrm{Pd}(100)$ for the three-layer situation (dotted line) into three shifted $2 p$ components (full line) and the total spectrum (full line). 
TABLE I. Measured interface and adhesion core level shifts in $\mathrm{eV}$ for $\mathrm{Na}, \mathrm{K}, \mathrm{Rb}$, and $\mathrm{Cs}$ on the indicated metals.

\begin{tabular}{lcc}
\hline \hline & $\Delta E_{\text {int }}$ & $\Delta E_{\text {adh }}$ \\
\hline $\mathrm{Na} / \mathrm{Mo}(110)$ & -0.41 & -0.63 \\
$\mathrm{Na} / \mathrm{Rh}(111)$ & -0.52 & -0.75 \\
$\mathrm{Na} / \mathrm{Pd}(100)$ & -0.72 & -0.93 \\
$\mathrm{~K} / \mathrm{Rh}(111)$ & -0.76 & -0.98 \\
$\mathrm{~K} / \mathrm{Pd}(100)$ & -0.96 & -1.16 \\
$\mathrm{Rb} / \mathrm{Rh}(111)$ & -0.77 & -0.98 \\
$\mathrm{Cs} / \mathrm{Mo}(110)$ & -0.78 & -0.98 \\
$\mathrm{Cs} / \mathrm{Rh}(111)$ & -1.07 & -1.28 \\
$\mathrm{Cs} / \mathrm{Pd}(100)$ & -1.16 & -1.37 \\
\hline
\end{tabular}

Changes of the lifetime are expected to be stronger for core holes that predominantly decay via channels which involve valence electrons. As other broadening mechanisms, disorder and vibrational, are expected to be similar for all core levels, the importance of lifetime changes may be deduced from measurements of the increase in width of different core levels of the alkali atom. From such measurements we find that for interface alkali atoms significant changes of the lifetime width occur for the outermost alkali $p$ core levels. The broadening of the one-layer peak in Fig. 1 is presumably due to the same effects. The slightly larger width of the surface peak compared to the bulk peak is in general explained by disorder and larger vibrational freedom in the surface than in the bulk. ${ }^{22,23}$ Substrate-induced broadening effects in alkali core levels are discussed in a separate paper. ${ }^{24} \mathrm{We}$ note that in the present context the main influence of this extra broadening is to decrease slightly the precision of the shifts which involve the interface peak. The application of a curve-fitting procedure similar to that shown in Fig. 4 to the alkali core-level spectra in Figs. 1-3 and additional spectra not shown results in the binding-energy shifts given in Table I. We estimate the measured binding-energy shifts to be accurate within $\pm 15 \mathrm{meV}$ for the bulk and surface peak and $\pm 30 \mathrm{meV}$ for the interface peak.

We define the interface shift $\Delta E_{\text {int }}$ as

$$
\Delta E_{\mathrm{int}}=E_{\mathrm{int}}^{B}-E_{\mathrm{bulk}}^{B},
$$

and the adhesion shift $\Delta E_{\text {adh }}$ as

$$
\Delta E_{\mathrm{adh}}=E_{\mathrm{int}}^{B}-E_{\mathrm{surf}}^{B},
$$

where $E_{\text {surf }}^{B}, E_{\text {bulk }}^{B}$, and $E_{\text {int }}^{B}$ are the core-level binding energies for the atoms at the surface, bulk, and interface, respectively.

\section{DISCUSSION}

Before discussing the observed trends we will address the fact that one of the substrate surfaces in Table I, namely, $\operatorname{Pd}(100)$, is not the most close-packed surface. It is well known that the structural properties of the substrate and the adsorbate at the interface must be taken into account when considering a core-level binding energy. ${ }^{2,4,6,25}$ To illustrate the magnitude of the changes which may result from a different structure of the substrate surface, we may compare measurements performed on the $R h(110)$ and the $R h(111)$ surfaces. In Table II, we present the measured interface and adhesion shifts for $\mathrm{K}$, $\mathrm{Rb}$, and $\mathrm{Cs}$ on $\mathrm{Rh}(111)$ and $\mathrm{Rh}(110)$. The more open (110) surface is expected to induce larger shifts of the alkali core levels, since the alkali atoms will experience a higher coordination to the $\mathrm{Rh}$ atoms at the (110) surface than at the (111) surface. As can be seen from Table II, this expectation is borne out by the experiments. For all the alkali metals the binding energy of the interface peak on the (110) surface is approximately $0.25 \mathrm{eV}$ lower than on the (111) surface. The rigid shift of $-0.25 \mathrm{eV}$ means that the trend (see Fig. 2) in the alkali interface shifts seen on the $R h(111)$ surface is still preserved on the $\mathrm{Rh}(110)$ surface. Based on this we expect that for any $4 d$ substrate the ordering of the alkali interface shifts will be independent of which particular surface is used. However, the lowering of the interface binding energy by 0.25 $\mathrm{eV}$ is of similar magnitude to comparing two different $4 d$ metal substrates. Since the $(100)$ surface is more close packed than the (110) surface, we would expect that the use of the Pd(100) instead of the Pd(111) surface could increase the alkali interface shifts by a similar amount. Thus a major part of the increase in the alkali interface shifts between Rh and Pd (see Fig. 3 and Table I) is likely to be due to the use of a more open surface for Pd.

It is well known that core-level binding-energy shifts in metallic systems can be interpreted in terms of various thermodynamical quantities. Two main requirements in this procedure for describing the shifts are the assumption of a fully screened final state and the $Z+1$ approximation for the screening valence charge distribution around the core ionized site. ${ }^{26}$ Under these assumptions a connection can be made between the core-level binding-energies and various thermodynamical properties of the systems. However, since photoemission is a vertical excitation process there is no geometric relaxation of the nuclear positions in the photoemission process whereby the thermodynamical properties should properly refer to such a geometrical nonrelaxed situation. This is a particular problem in the case of alkali metals (and for divalent lanthanide elements, see Ref. 25) since here the atomic volumes between the $Z$ and $Z+1$ atoms differ significantly. We will address this problem further below. In most cases however, the difference in atomic volume between the $Z$ and the $Z+1$ metal is quite small, resulting in negligible modifications of the shifts. Using this thermodynamical approach, which takes into account both the initial state and the final state, chemical shifts in

TABLE II. Measured interface and adhesion core-level in eV for $\mathrm{K}, \mathrm{Rb}$, and $\mathrm{Cs}$ on $\mathrm{Rh}(111)$ and $\mathrm{Rh}(110)$.

\begin{tabular}{lcccc}
\hline & \multicolumn{2}{c}{$\Delta E_{\text {int }}$} & \multicolumn{2}{c}{$\Delta E_{\text {adh }}$} \\
& $\mathrm{Rh}(111)$ & $\mathrm{Rh}(110)$ & $\mathrm{Rh}(111)$ & $\mathrm{Rh}(110)$ \\
\hline $\mathrm{K}$ & -0.76 & -1.03 & -0.98 & -1.24 \\
$\mathrm{Rb}$ & -0.77 & -1.03 & -0.98 & -1.25 \\
$\mathrm{Cs}$ & -1.07 & -1.32 & -1.28 & -1.53 \\
\hline \hline
\end{tabular}


several different types of systems such as surfaces, ${ }^{16,26}$ alloys, ${ }^{27,28}$ and interfaces ${ }^{9,16,25}$ have been successfully treated.

It has been shown elsewhere ${ }^{9}$ by using the assumptions mentioned above and neglecting the lattice relaxation problem that the shifts in the present type of systems may be interpreted in terms of segregation and adhesion energies. The interface shift, i.e., the binding-energy shift between bulk and interface atoms, of a metal $Z$ is equal to the interface segregation energy of a $Z+1$ impurity from the bulk of the $Z$ metal to the interface. ${ }^{9}$ From the experimental values in Table $I$ it is found that $Z+1$ impurities in the alkali layers will segregate to any of the $4 d$ metal interfaces, with increasingly higher interface segregation energy going from $\mathrm{Na}$ to $\mathrm{K}$ to $\mathrm{Rb}$ to Cs. It can also be seen from Table $I$ that when changing the substrate from $\mathrm{Mo}$ to $\mathrm{Ru}$ to $\mathrm{Rh}$ to $\mathrm{Pd}$ the interface segregation energy increases for the $Z+1$ impurity in the alkali layers. If we compare the measured binding-energy shifts presented here with the measured shifts from alkali films on $s p$ metals ${ }^{16-18}$ we see that in general the shifts are larger in the present case. This means that the interface segregation energy is larger for a $Z+1$ atom in an alkali film on a $4 d$ metal than on an $s p$ metal, thus the $Z+1 \mathrm{im}$ purity is more strongly bound at an interface towards a $4 d$ metal than at one towards an $s p$ metal.

The adhesion shift, i.e., the binding-energy shift between interface and surface atoms of a metal with atomic number $Z$ is related to the difference in adhesion energy of the $Z$ and of the $Z+1$ metals on the same substrate ${ }^{9}$ (see below for a more detailed discussion of this point). The sign of the adhesion shifts directly shows that the adhesion energy on $4 d$ metals is larger for the alkaline earth metals than for the alkali metals. This difference increases as we go from $\mathrm{Na}$ to $\mathrm{K}$ to $\mathrm{Rb}$ to Cs deposited on the same $4 d$ metal substrate and furthermore also increases with increasing atomic number of the $4 d$ metal substrate. Comparing to alkali metals on $s p$ metals ${ }^{16-18}$ we find that the difference in adhesion energy for the $Z+1$ (alkaline earth) and the $Z$ (alkali) metal is larger for a $4 d$ metal substrate than for an $s p$-metal substrate.

Apart from allowing us to obtain values for these thermodynamical quantities from the core-level shifts, which is difficult by other methods, these relationships also make it possible to estimate the core-level shifts from various thermodynamical parameters. Since experimental values do not exist for all the relevant thermodynamical parameters, we calculate these by Miedema's semiempirical scheme ${ }^{29,30}$ which may introduce some uncertainties. Apart from this, additional problems may, as discussed above, be caused by the use of values (calculated or experimental) for these thermodynamical parameters which refer to a geometrically relaxed situation and not to the nonrelaxed situation relevant for core-level photoemission.

Here we use a model to estimate the interface shift which is based on segregation energies, which may be calculated using Miedema's semiempirical scheme. ${ }^{29}$ The model used to estimate the adhesion shift is based on adhesion energies, which also may be calculated using Miedema's scheme. ${ }^{30} \mathrm{~A}$ detailed description of the two models may be found in Ref. 9. A third and somewhat more general model based on a decomposition of the shifts into partial shifts ${ }^{19,31}$ may be used to calculate both the interface and the adhesion shift. Before coming to the actual calculated values for the shifts we will discuss two main complications encountered in these calculations. This discussion is of course relevant also for the extraction of thermodynamical values from the experimentally determined core-level binding-energy shifts, as presented above.

The first complication is related to Miedema's scheme itself and may be illustrated by the calculations of adhesion energies. By definition the adhesion energy per area of metal $A$ on metal $B$ should be equal to that of $B$ on $A$. Miedema's expression for the adhesion energy ${ }^{30}$ does not always yield this symmetry. This is due to the so-called chemical term $\gamma^{\text {chem }}$, which numerically often turns out to be different for $A$ on $B$ and $B$ on $A$, respectively, although it actually should be equal according to Miedema and Den Broeder. ${ }^{36}$ To make the formalism symmetric we use, as recommended by Miedema and Den Broeder, ${ }^{36}$ the average value of the chemical term for the two cases, that is, $\gamma^{\text {chem }}=\left(\gamma_{A \text { on } B}^{\text {chem }}+\gamma_{B \text { on } A}^{\text {chem }}\right) / 2$. $\mathrm{Cs}$ and $\mathrm{Ba}$ on Mo may illustrate how much this procedure will influence the estimated adhesion energy difference. Using the average value for the chemical term when calculating the adhesion energy of Cs on Mo yields a value of $-1.26 \mathrm{~J} / \mathrm{m}^{2}$, while using only the value for Cs on Mo gives $-1.68 \mathrm{~J} / \mathrm{m}^{2}$. In the case of $\mathrm{Ba}$ we arrive at corresponding values of -1.89 and $-2.07 \mathrm{~J} / \mathrm{m}^{2}$, respectively. This will then result in adhesion energy differences between the $Z+1(\mathrm{Ba})$ and the $Z(\mathrm{Cs})$ atom for the two cases of -0.63 and $-0.39 \mathrm{~J} / \mathrm{m}^{2}$. These values would (see below for details) result in adhesion shifts of -1.01 and $-0.63 \mathrm{eV}$, respectively, which should be compared to the experimentally obtained value of $-0.98 \mathrm{eV}$. By performing these calculations for all the systems presented in this paper we conclude that using the average value for the chemical term is the most appropriate assumption when calculating the adhesion energies for the present systems, a conclusion which is consistent with the recommendation of Miedema and Den Broeder. ${ }^{36}$ The same chemical term is also present in Miedema's expression for the interface segregation energies. ${ }^{29}$ Thus, following the recommendation of Miedema and Den Broeder, ${ }^{36}$ we also use the average value for the chemical term when calculating the interface segregation energies. The procedure of using the average value of the chemical term was not applied when estimating the layer-resolved shifts in the alkali-Al interface systems. ${ }^{16}$

The second complication is related to the fact that the difference in atomic volume in the case of alkali atoms between the $Z$ and $Z+1$ atom is appreciable. First, as mentioned above, there is no geometrical relaxation of the nuclear positions in the photoemission process meaning that the calculations of adhesion and segregation energies should be performed for $Z+1$ atoms having the size of the $Z$ atoms, which is not possible using Miedema's scheme. This problem is commonly encountered in thermodynamical estimates of core-level binding-energy shifts, see, e.g., Ref. 25 , and is normally 
ignored because the effects are considered to be relatively small. ${ }^{9,16,25-28}$ The present results (see below) indicate that this is also the case for the present systems despite the large size difference between $Z$ and $Z+1$. Second, the large size difference makes it necessary to consider how the adhesion shift $\Delta E_{\text {adh }}$ should be related to the adhesion energy per area which is the natural dimension of an adhesion energy. In Ref. 9 it was argued that the adhesion shift is equal to the adhesion energy difference per atom of the $Z$ and the $Z+1$ metal on the substrate. However, from the derivation of this relation in Ref. 9 it seems to be more natural to use the adhesion difference per interface area of a $Z$ atom instead of per atom of $Z$ and $Z+1$. This is a way of taking into account the fact that in the photoemission process the final-state $Z+1$ atom has the size of a $Z$ atom.

If one wants to preserve the statement that the adhesion shift $\Delta E_{\mathrm{adh}}$ is the adhesion difference per atom, then the adhesion energy for the $Z+1$ metal should be calculated using the lattice constant of the $Z$ metal. This is not possible in Miedema's scheme. In fact, this is a minor complication in most cases due to the normally similar size of the $Z$ and the $Z+1$ atoms and the problem only becomes severe when the size difference is appreciable as in the present systems. By calculating the adhesion energy difference per atom and per interface area of the $Z$ atom, and comparing to the measured shifts we find that the adhesion energy difference per $Z$ area gives by far the best agreement. $\mathrm{Cs}$ and $\mathrm{Ba}$ on Mo may be used to illustrate the effects of using the adhesion energy difference either per atom of $Z$ and $Z+1$ or per interface area of the $Z$ atom. Assuming close-packed Cs and $\mathrm{Ba}$ interface layers on the Mo surface ${ }^{37}$ and using the values given above yields adhesion energies per atom of -2.03 and $-2.04 \mathrm{eV}$ and per $Z$ atom interface area of -2.03 and $-3.04 \mathrm{eV}$ for $\mathrm{Cs}$ and $\mathrm{Ba}$, respectively. By comparison to the experimental value for the adhesion shift $(-0.98 \mathrm{eV})$ we see that much better agreement is found for the adhesion energy difference using the $Z$ atom interface area $(-1.01 \mathrm{eV})$ than using the adhesion energy difference per atom of $Z$ and $Z+1(-0.01 \mathrm{eV})$. By performing this comparative procedure for all the systems presented in this paper we conclude that the most appropriate procedure is to use the adhesion energies per $Z$ atom interface area when relating the adhesion energy difference to the adhesion shift $\Delta E_{\text {adh }}$ measured by corelevel photoemission. ${ }^{38}$

In Fig. 5(a) we present a comparison between the experimental values for the interface shift $\Delta E_{\text {int }}$ for $\mathrm{Na}, \mathrm{K}$, and $C s$ on the four different $4 d$ metals, and the values from the two different models based on segregation energies and partial shifts. In Fig. 5(b) a comparison is presented between the experimental values for the adhesion shift and the values calculated from the two different models based on adhesion energies and partial shifts. From Figs. 5(a) and 5(b) we can see that the calculated values reproduce the experimentally obtained values reasonably well. It is particularly gratifying to note that the models produce a negative shift of the alkali corelevel binding energies. All simple initial-state models relating the shifts to charge transfer would yield a positive
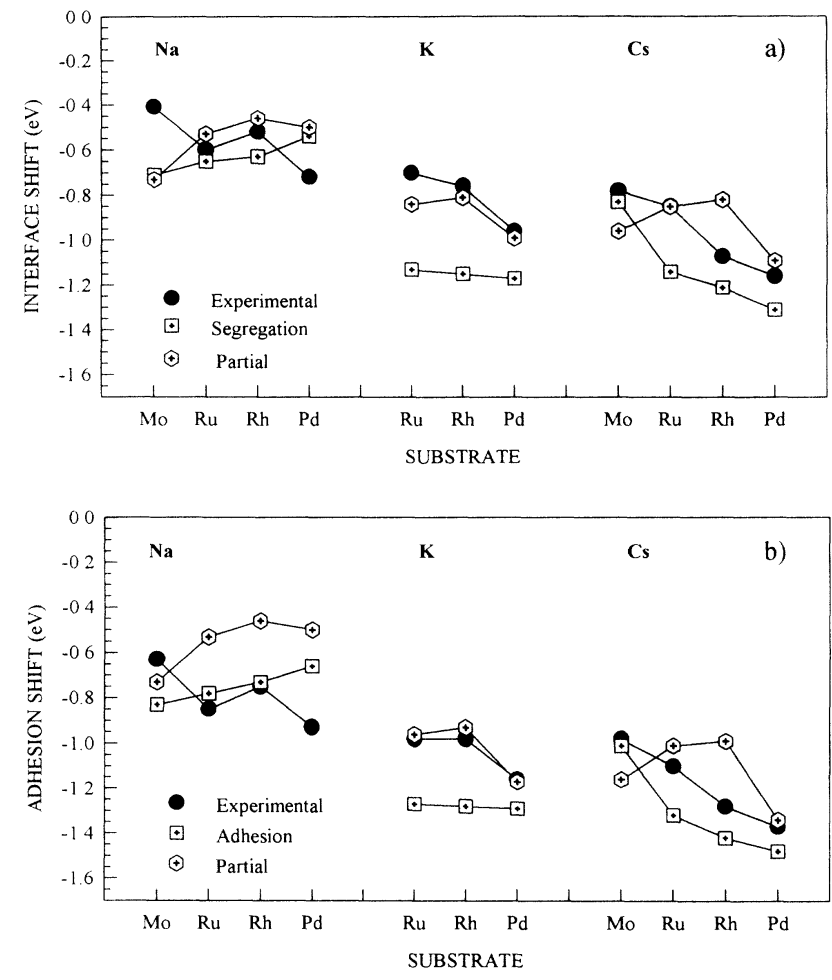

FIG. 5. (a) Measured and calculated interface shifts for $\mathrm{Na}$, $\mathrm{K}$, and $\mathrm{Cs}$ on the indicated metals. The measured values of $\mathrm{Na}$. $\mathrm{K}$, and $\mathrm{Cs}$ on $\mathrm{Ru}(100)$ were taken from Refs. 13 and 14. See also Ref. 39. (b) Measured and calculated adhesion shifts for $\mathrm{Na}, \mathrm{K}$, and $\mathrm{Cs}$ on the indicated metals. The measured values of $\mathrm{Na}, \mathrm{K}$, and $\mathrm{Cs}$ on $\mathrm{Ru}(100)$ were taken from Refs. 13 and 14. See also Ref. 39.

shift of the alkali core-level binding energies in these systems, unless of course one makes the unrealistic assumption that electrons are transferred from the substrate to the alkali making the alkali interface layer negatively charged. Concerning the magnitudes and the trends, with respect to alkali as well as to $4 d$ metal, of the alkali core-level binding-energy shifts we find that these are reasonably well reproduced by the thermodynamical models, in particular when considering the complications discussed above and the approximative nature of Miedema's scheme.

In summary we have measured layer-resolved corelevel binding-energy shifts for $\mathrm{Na}, \mathrm{K}, \mathrm{Rb}$, and $\mathrm{Cs}$ on Mo(110), $R$ h(111), $R h(110)$, and $\operatorname{Pd}(100)$ by highresolution core-level spectroscopy. We have obtained information on interface segregation and adhesion energies. The experimental results can be reproduced well by models based on a thermodynamical description using as input parameters values calculated by Miedema's scheme. This, however, requires that the large change in size between an alkali atom and the next element in the Periodic Table is taken into account.

\section{ACKNOWLEDGMENT}

Financial support of this work by the Swedish Natural Science Research Council is gratefully acknowledged. 
${ }^{1}$ Physics and Chemistry of Alkali Metal Adsorption, edited by $\mathbf{H}$. P. Bonzel, A. M. Bradshaw, and G. Ertl (Elsevier, Amsterdam, 1989).

2J. N. Andersen, E. Lundgren, R. Nyholm, and M. Qvarford, Surf. Sci. 289, 307 (1993).

${ }^{3}$ A. Schmalz, S. Aminpirooz, L. Becker, J. Haase, J. Neugebauer, M. Scheffler, D. R. Batchelor, D. L. Adams, and E. Bøgh, Phys. Rev. Lett. 67, 2163 (1991).

${ }^{4}$ J. N. Andersen, M. Qvarford, R. Nyholm, J. F. van Acker, and E. Lundgren, Phys. Rev. Lett. 68, 94 (1992).

${ }^{5}$ C. Stampfl, M. Scheffler, H. Over, J. Burchhardt, M. M. Nielsen, D. L. Adams, and W. Moritz, Phys. Rev. Lett. 69, 1532 (1992).

6J. N. Andersen, E. Lundgren, R. Nyholm, and M. Qvarford, Phys. Rev. B 46, 12784 (1992).

${ }^{7} \mathrm{~J}$. Neugebauer and M. Scheffler, Phys. Rev. Lett. 71, 577 (1993).

${ }^{8}$ X. Shi, D. Tang, D. Heskett, K.-D. Tsuei, H. Ishida, and Y. Morikawa, Surf. Sci. 290, 69 (1993).

${ }^{9}$ N. Mårtensson, A. Stenborg, O. Björneholm, A. Nilsson, and J. N. Andersen, Phys. Rev. Lett. 60, 1731 (1988).

${ }^{10}$ A. Hohlfeld, M. Sunjic, and K. Horn, J. Vac. Sci. Technol. A 5, 679 (1987).

${ }^{11}$ A. Stenborg, O. Björneholm, A. Nilsson, N. Mårtensson, J. N. Andersen, and C. Wigren, Surf. Sci. 211/212, 470 (1989).

12J. N. Andersen, O. Björneholm, A. Stenborg, A. Nilsson, C. Wigren, and N. Mårtensson, J. Phys. Condens. Matter 1, 7309 (1989).

${ }^{13}$ M.-L. Shek, J. Hrbek, T. K. Sham, and G.-Q. Xu, Phys. Rev. B 41, 3447 (1990).

${ }^{14}$ T. K. Sham, G.-Q. Xu, J. Hrbek, and M.-L. Shek, Surf. Sci. 210, L185 (1989).

${ }^{15}$ D. M. Riffe, G. K. Wertheim, and P. H. Citrin, Phys. Rev. Lett. 64, 571 (1990).

${ }^{16}$ E. Lundgren, J. N. Andersen, M. Qvarford, and R. Nyholm, Surf. Sci. 281, 83 (1993).

${ }^{17}$ E. Lundgren, J. N. Andersen, M. Qvarford, and R. Nyholm, MAX-LAB Activity Report (1991).

${ }^{18}$ E. Lundgren, R. Nyholm, and J. N. Andersen (unpublished).

${ }^{19}$ J. N. Andersen, O. Björneholm, A. Sandell, R. Nyholm, J. Forsell, L. Thånell, A. Nilsson, and N. Mårtensson, Synchrotron Radiat. News 4, 15 (1991).

${ }^{20}$ R. Nyholm, S. Svensson, J. Nordgren, and S. A. Flodström, Nucl. Instrum. Methods A 246, 267 (1986).

${ }^{21}$ SAES Getters, SpA, Milano, Italy.

${ }^{22}$ A. Stenborg, J. N. Andersen, O. Björneholm, A. Nilsson, and N. Mårtensson, J. Electron. Spectrosc. Relat. Phenom. 52, 47 (1990).

${ }^{23}$ D. M. Riffe, G. K. Wertheim, and P. H. Citrin, Phys. Rev. Lett. 67, 116 (1991).

${ }^{24} \mathrm{~J}$. N. Andersen, E. Lundgren, and R. Nyholm (unpublished).

${ }^{25}$ A. Nilsson, B. Eriksson, N. Mårtensson, J. N. Andersen, and J. Onsgaard, Phys. Rev. B 38, 10357 (1988).

${ }^{26}$ B. Johansson and N. Mårtensson, Phys. Rev. B 21, 4427 (1980).

${ }^{27}$ N. Mårtensson, R. Nyholm, H. Calén, J. Hedman, and B. Johansson, Phys. Rev. B 24, 1725 (1981).

${ }^{28}$ P. Steiner, S. Hüfner, N. Mårtensson, and B. Johansson, Solid State Commun. 37, 73 (1981); P. Steiner and S. Hüfner, Acta Metall. 29, 1885 (1981).
${ }^{29}$ The interface segregation energy can be written as the sum of the three terms of solution and surface energies (Ref. 32). We have used surface energies from Ref. 33 and solution energies from Ref. 34.

${ }^{30}$ The adhesion energy in Miedema's formalism (Ref. 32) is given by

$$
E_{\mathrm{adh}}=-\gamma_{M}-\gamma_{\mathrm{Z}}+\gamma^{\mathrm{chem}}+\gamma_{\mathrm{mm}},
$$

where $\gamma_{M}$ and $\gamma_{Z}$ are the surface energies for the pure metals, $\gamma^{\text {chem }}$ and $\gamma_{\mathrm{mm}}$ are the contributions from the interfacial energy $\gamma_{M, Z}$, in which $\gamma^{\text {chem }}$ is related to the energy of alloying and $\gamma_{m m}$ is the average grain boundary mismatch energy. The mismatch energy has been chosen to be $15 \%$ of the sum of the surface energies (Ref. 32). We have used surface energies from Ref. 33 and solution energies from Ref. 34.

${ }^{31}$ In the partial shift model we have to restrain the different energy parameters by some choice of effective concentration parameters describing to what extent each type of neighbor is present around the ionized atom (Ref. 25). These effective concentration parameters are chosen so that $C_{Z}^{*}=0.75$ at the interface and the surface and 1 in the bulk of the $Z$ metal, $C_{M}^{*}=0.25$ at the interface and 0 at the surface and in the bulk, and $C_{V}^{*}=0.25$ at the surface and 0 at the interface and in the bulk for the close-packed surfaces. In the case of the more open $\operatorname{Pd}(100)$ surface the choice is instead $C_{Z}^{*}=0.65$ at the interface and the surface and 1 in the bulk of the $Z$ metal, $C_{M}^{*}=0.35$ at the interface and 0 at the surface and in the bulk, and $C_{V}^{*}=0.35$ at the surface and 0 at the interface and in the bulk. For the cohesive energies see Ref. 35.

32J. Gerkema and A. R. Miedema, Surf. Sci. 124, 351 (1983).

${ }^{33}$ H. L. Skriver and N. M. Rosengard, Phys. Rev. B 46, 7157 (1992).

${ }^{34}$ A. R. Miedema, F. R. de Boer, and R. Boom, CALPHAD Comput. Coupling Phase Diagrams Thermochem. 1, 341 (1977).

${ }^{35}$ C. Kittel, Introduction to Solid State Physics, 6th ed. (Wiley, New York, 1986), p. 55.

${ }^{36}$ A. R. Miedema and F. J. A. Den Broeder, Z. Metallkd. 70, 14 (1979).

${ }^{37}$ For all the alkali metals we use a size corresponding to the pure alkali metal and thus neglect the compression of the alkali-alkali distance often found for the first adsorbed alkali layer. Inclusion of this compression would typically reduce the calculated shifts by 5-10\%.

${ }^{38}$ For the case of alkali metals on Al (Ref. 16) we did not use this procedure but instead related the adhesion shift to the adhesion energy difference per atom. The reason why we still obtained good agreement may be traced back to the fact that the surface energies entering into the calculations of adhesion energies were taken from Miedema's calculations. If we instead, as in the present paper, use surface energies calculated by $a b$ initio methods (Ref. 33 ), we find that the agreement is improved if instead the area per $Z$ atom is used.

${ }^{39}$ The values for $\mathrm{Na}$ and $\mathrm{Cs}$ on $\mathrm{Ru}(100)$ are taken from values given in Ref. 13 whereas the values for $\mathrm{K}$ on $\mathrm{Ru}(100)$ are taken from values given in Ref. 14. In the case of Cs on $R u(100)$, measurements have been performed using either the Cs $5 p$ (Ref. 14) or the Cs $4 d$ (Ref. 13) core level. We have used the value for the Cs $4 d$ level. 